\title{
Synthesis and Antibacterial Activity of Antibiotic-Functionalized Graphite Nanofibers
}

\author{
Madeline Rotella, Alicia Briegel, John Hull, Anthony Lagalante, and Robert Giuliano \\ Department of Chemistry, Villanova University, Villanova, PA 19085, USA \\ Correspondence should be addressed to Robert Giuliano; robert.giuliano@villanova.edu
}

Received 14 November 2014; Accepted 28 January 2015

Academic Editor: Nurit Beyth

Copyright (C) 2015 Madeline Rotella et al. This is an open access article distributed under the Creative Commons Attribution License, which permits unrestricted use, distribution, and reproduction in any medium, provided the original work is properly cited.

Surface functionalization of nanomaterials is an area of current investigation that supports the development of new biomaterials for applications in biology and medicine. Herein we describe the synthesis, characterization, and antibacterial properties of the first examples of antibiotic-labeled graphitic carbon nanofibers (GCNFs) covalently functionalized with aminoglycoside and quinolone antibiotics. Ruthenium tetroxide oxidation of herringbone GCNFs gave higher amounts of surface carboxyl groups than previous methods. These carboxyl groups served as sites of attachment for antibiotics by acyl substitution. Bioassay of these novel, functionalized GCNFs using serial dilution and optical density methods demonstrated that antibiotic-labeled GCNFs possess significant antibacterial activity against Pseudomonas aeruginosa. The activity we observe for aminoglycoside-functionalized GCNFs suggests a membranolytic mechanism of action.

\section{Introduction}

Graphite carbon nanofibers (GCNFs) are novel nanoscale materials that can be prepared inexpensively, in multigram quantities, via the decomposition of carbon monoxide or hydrocarbons over mono- or bimetallic catalysts [1-4]. Three types of crystalline GCNFs can be obtained, designated as ribbons, platelets, or herringbones (Figure 1). A TEM image of as-produced ribbon GCNFs is shown in Figure 2.

Each of the GCNF structures maintains a minimum interlayer spacing of $0.34 \mathrm{~nm}$, which corresponds to crystalline graphite. The width of the nanofibers is in the range of 5$500 \mathrm{~nm}$, depending on the growth catalyst and fiber morphology, while they vary in length from 5 to $100 \mu \mathrm{m}$ and in surface area from 50 to $350 \mathrm{~m}^{2} / \mathrm{gm}$. The unique structures and physical properties of GCNFs and their low cost of production compared to CNTs have generated interest in their use in a wide range of applications. Initial applications of GCNFs focused on their absorption and storage capacities and led to the development of GCNFs as adsorbents for small organic molecules from aqueous streams [5], electrodes for fuel cells [6], hydrogen storage media [7], and heterogeneous catalyst supports [8]. More recently, the well-recognized potential of carbon nanomaterials, especially those that contain surface functionality, has resulted in a broader range of interest in the application of graphite nanofibers in biology, medicine, composite materials, and energy conversion. In particular, promising studies have been described in which functionalized GCNFs have been investigated as biosensors [9], biomimetic membranes [10], neuroelectrochemical electrodes [11], and scaffolds for neural tissue regeneration and drug delivery [12]. GCNF composite materials have also been evaluated as solid state gas sensors [13].

In spite of these advances, there remain comparatively few published studies of the surface functionalization of graphite nanofibers with biologically active ligands, especially when compared to the development of functionalized fullerenes and carbon nanotubes [14-17]. We are particularly interested in the covalent attachment of biologically active carbohydrates to the surface of graphite nanofibers. Since carbohydrates are enormously rich in terms of their diversity of structure and their biological functions, the potential exists for the development of carbohydrate-functionalized GCNFs with a vast range of properties and potential as biomaterials. 


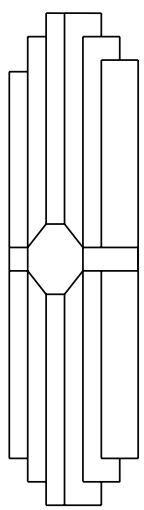

(a)

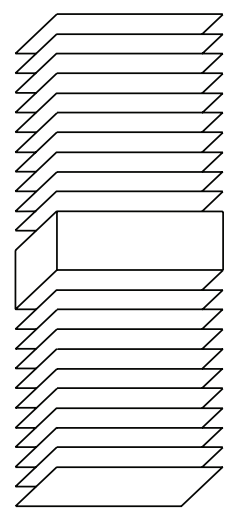

(b) (c)

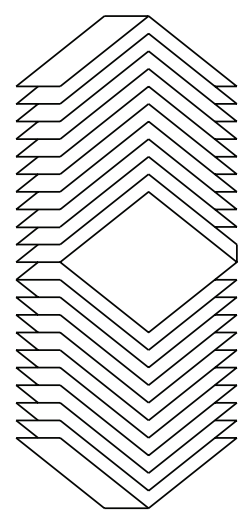

FIGURE 1: GCNF structures: (a) ribbon; (b) platelet; (c) herringbone.

Graphitic materials such as pyrolytic and turbostratic carbon have been used previously as components in the fabrication of medical devices such as heart valves and stents [18]. However, one of the problems associated with some of these devices is infection that results from the attachment and colonization of bacteria on the surface leading to biofilm formation $[19,20]$. Improved biocompatibility may be addressed through surface modification with antibiotics that may prevent the initial attachment of bacteria [21]. For this study, we chose GCNFs functionalized with carbohydrate aminoglycoside antibiotics and a noncarbohydrate antibiotic as our target structures, in an effort to determine whether antibacterial properties could be conferred to the GCNF scaffold by covalent labeling. Given the dimensions of the GCNFs and that fact that the biological target for aminoglycoside antibiotics is normally intracellular, it could not be assumed that the antibiotics would function as biocidal agents in the surface-bound state. However, a study in which it was shown that nanoparticles labeled with gentamicin were active against $P$. aeruginosa suggested that alternative mechanisms of action may be accessible to the aminoglycoside class of antibiotics [22]. In addition, a composite made of functionalized SWNTs covalently bound to a polyamide membrane was active against $E$. coli, and a hybrid material made of MWNTs with a coating of immobilized amoxicillin was active against E. coli and S. aureus $[23,24]$. These studies suggest that new biomaterials could be based on the GCNF scaffold. In this paper, we describe the first covalent functionalization of oxidized graphite nanofibers with the aminoglycoside antibiotics tobramycin and amikacin and with the fluoroquinolone antibiotic ciprofloxacin. Methods are described for the synthesis and characterization of these novel antibiotic-labeled GCNFs, and results of biological testing against the pathogenic organism Pseudomonas aeruginosa are presented.

\section{Materials and Methods}

2.1. Production and Characterization of Graphite Nanofibers. Herringbone graphite nanofibers were prepared in a Thermolyne 79400 tube furnace equipped with Omega FMA $5400 / 5500$ mass flow controllers for $\mathrm{C}_{2} \mathrm{H}_{4}$, air, $\mathrm{He}$, and $\mathrm{H}_{2}$

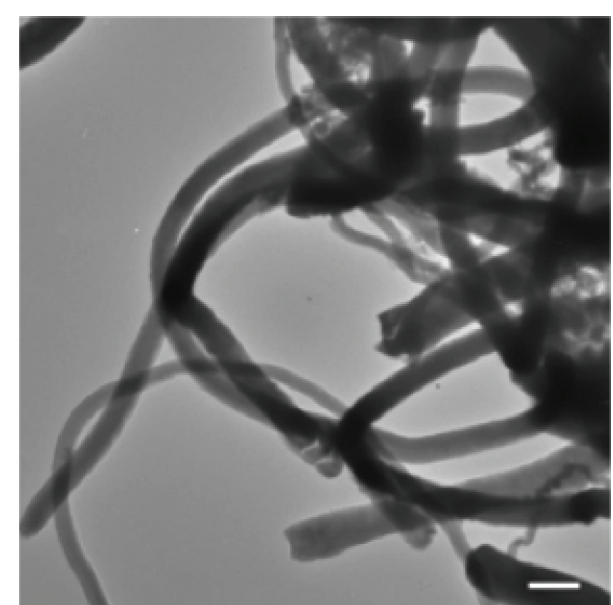

FIGURE 2: TEM of herringbone GCNFs (scale bar, lower right = $500 \mathrm{~nm})$.

[1-4]. Herringbone GCNFs were produced using a bimetallic copper-nickel catalyst. From $50 \mathrm{mg}$ of copper-nickel catalyst $6.73 \mathrm{~g}$ of herringbone GCNFs was obtained. TEM and SEM images were obtained using a Hitachi $\mathrm{H}-7600$ electron microscope (lattice resolution $=0.204 \mathrm{~nm}$ ) and a Hitachi S-570 field-emission-scanning electron microscope, respectively. For TEM, the fibers were dissolved in isopropanol and a drop was placed on a TEM grid and dried overnight. The microscope was operated at an electron accelerating voltage of $100.0 \mathrm{kV}$ high resolution and images were obtained at magnifications of 4000x, 8000x, 15000x, 20000x, 40000x, and 60000x. For SEM, the fibers were mounted on carbon adhesives, dried overnight, and then sputter-coated with gold for 90 seconds using an EMS150RES Quorum Sputter Coating system. The microscope was operated at an electron accelerating voltage $5.0 \mathrm{kV}$ and a $15 \mathrm{~mm}$ working distance. Images obtained at magnifications of $1000 \mathrm{x}$ and 5000x are shown in Figure 6.

2.2. $\mathrm{RuO}_{4}$ Oxidation of GCNFs. The procedure of Rasheed et al. was followed with modifications [25]. Activated ruthenium dioxide [26] (40 mg) was added to commercial bleach $(300 \mathrm{~mL})$ and the mixture was stirred for $10 \mathrm{~min}$ at room temperature, producing a yellow solution of $\mathrm{RuO}_{4}$. As-produced herringbone GCNFs (1.0 g) were added and the mixture was stirred for 3 days. At the end of the first and second days, additional bleach $(40 \mathrm{~mL})$ was added to the mixture. After 3 days, the mixture was filtered through a $0.45 \mu \mathrm{m}$ nylon membrane and washed with $100 \mathrm{~mL}$ of distilled water in small portions. The fibers were dried under reduced pressure (25 Torr) at $40^{\circ} \mathrm{C}$ for one day in a benchtop oven, resulting in a yield of $760 \mathrm{mg}$. Surface analysis of $\mathrm{RuO}_{4}$-oxidized GCNFs was carried out by XPS.

2.3. Tobramycin Labeling of $\mathrm{RuO}_{4}$-Oxidized GCNFs by Reductive Amination. To a stirring suspension of $\mathrm{RuO}_{4}$-oxidized herringbone GCNFs $(24.5 \mathrm{mg})$ in THF $(125 \mathrm{~mL}$, reagent grade) $35.0 \mathrm{~mL}$ of a $100 \mu \mathrm{M}$ solution of tobramycin $(11.7 \mathrm{mg}$, 
$0.025 \mathrm{mmol}$, diluted to $250 \mathrm{~mL}$ THF) in THF and $100.0 \mu \mathrm{L}$ triethylamine (Aldrich, 99.5\%) was added. The mixture was stirred for 2 days at room temperature. After $48 \mathrm{~h}$, sodium borohydride was added $(10 \mathrm{~mL}$ of a $200 \mu \mathrm{M}$ in solution in THF) and the mixture was stirred for $24 \mathrm{~h}$. The fibers were then collected by filtration through a $0.45 \mu \mathrm{m}$ nylon membrane filter and washed extensively with water until the $\mathrm{pH}$ of the filtrate was neutral. The fibers were placed in the oven at $80^{\circ} \mathrm{C}$ and 25 Torr to dry for 1 day to give tobramycinlabeled $\mathrm{RuO}_{4}$-oxidized GCNFs $(13.5 \mathrm{mg}$ ).

2.4. General Procedure for Antibiotic Labeling of $\mathrm{RuO}_{4}$-Oxidized GCNFs Using Thionyl Chloride Activation. The procedure of Pompeo and Resasco was followed [27]. To a $100 \mathrm{~mL}$ round-bottom flask containing $\mathrm{RuO}_{4}$-oxidized herringbone GCNFs (100 mg), $\mathrm{SOCl}_{2}$ (20.0 mL, Aldrich 99.5\%) and anhydrous DMF $(1.0 \mathrm{~mL})$ were added. The flask was equipped with a reflux condenser and a drying tube and the mixture was stirred at $65^{\circ} \mathrm{C}$ for $24 \mathrm{~h}$. The reaction mixture was cooled to room temperature and filtered through a $0.45 \mu \mathrm{m}$ membrane filter and washed with anhydrous THF $(3 \times 20 \mathrm{~mL})$. The fibers were dried under vacuum (25 Torr) for 1.5 hours and then suspended in THF $(30.0 \mathrm{~mL})$ with tobramycin $(20 \mathrm{mg}$, $0.043 \mathrm{mmol}$ ), amikacin ( $25 \mathrm{mg}, 0.043 \mathrm{mmol}$ ), or ciprofloxacin $(27 \mathrm{mg}, 0.081 \mathrm{mmol})$. The mixtures were stirred at $70^{\circ} \mathrm{C}$ under reflux for two days. The suspensions were cooled, washed with THF through a $0.45 \mu \mathrm{m}$ membrane filter, and dried overnight in the benchtop oven to give tobramycinlabeled GCNFs (40 mg), amikacin-labeled GCNFs (98 mg), or ciprofloxacin-labeled GCNFs $(78 \mathrm{mg}$ ). Each of the three samples of antibiotic-labeled GCNFs was washed successively with $\mathrm{HCl}(1 \mathrm{M}, 10 \mathrm{~mL}), \mathrm{dH}_{2} \mathrm{O}(10 \mathrm{~mL}), \mathrm{NaHCO}_{3}(1 \mathrm{M}, 10 \mathrm{~mL})$, and finally $\mathrm{dH}_{2} \mathrm{O}(10 \mathrm{~mL})$ using a $0.45 \mu \mathrm{m}$ nylon membrane filter mounted on a stainless steel screen in a solvent purification filter. The washes were collected and analyzed by mass spectrometry for unbound antibiotic. Antibiotic-labeled GCNF samples were redried overnight at $40^{\circ} \mathrm{C}$ and 25 Torr before biological testing.

2.5. X-Ray Photoelectron Spectroscopic Analysis of GNFs. XPS analyses were performed at Drexel University utilizing a PHI Versaprobe 5000 instrument equipped with a monochromated AIK alpha source. Analyses were carried out with a high power X-ray setting of 100 microns $25 \mathrm{~W}$ e-beam. Photoelectrons were collected using a hemispherical analyzer. Pass energies of $117 \mathrm{~V}$ and $23 \mathrm{~V}$ were used for survey and high resolution spectra. Samples were mounted using double-sided tape to the regular $2^{\prime \prime}$ sample holder. Both electron and iongun neutralizers were used during analysis to charge compensate the sample. Results of percent atom concentrations are shown in Table 2 (Results and Discussion).

2.6. Analysis of GCNFs for Unbound Antibiotic by Mass Spectrometry. Using flow injection tandem mass spectrometry (FI-MS/MS), the presence of all three antibiotics was monitored in the filtrates obtained during washing of the GCNFs with water and organic solvent. Individual multiple reaction
TABLE 1: Optimized compound potentials for the antibiotic MRM transitions and limits of detection.

\begin{tabular}{lccccc}
\hline Antibiotic & $\begin{array}{c}\text { Precursor } \\
(\mathrm{m} / \mathrm{z})\end{array}$ & $\begin{array}{c}\text { Fragment } \\
(\mathrm{m} / \mathrm{z})\end{array}$ & $\begin{array}{c}\mathrm{DP} \\
(\mathrm{V})\end{array}$ & $\begin{array}{c}\mathrm{CE} \\
(\mathrm{V})\end{array}$ & $\begin{array}{c}\text { LOD } \\
(\mathrm{ppb})\end{array}$ \\
\hline Amikacin & 586.2 & 163.2 & 20 & 47 & 89 \\
Ciprofloxacin & 332.4 & 231.4 & 50 & 54 & 1160 \\
Tobramycin & 468.4 & 163.2 & 17 & 35 & 146 \\
\hline
\end{tabular}

TABLE 2: XPS quantification of $\mathrm{RuO}_{4}$-oxidized and antibioticlabeled GCNFs.

\begin{tabular}{lccc}
\hline \multicolumn{1}{c}{ Sample } & C1s & $\begin{array}{c}\text { Binding } \\
\text { energy }(\mathrm{eV})\end{array}$ & $\begin{array}{c}\text { \%atom } \\
\text { concentration }\end{array}$ \\
\hline \multirow{4}{*}{ RuO $_{4}$-oxidized GCNFs } & $\mathrm{N} 1 \mathrm{~s}$ & 400.0 & 91.2 \\
& $\mathrm{O} 1 \mathrm{~s}$ & 532.5 & 0.0 \\
& $\mathrm{~S} 2 \mathrm{p}$ & 164.9 & 8.8 \\
\hline \multirow{4}{*}{ Amikacin-labeled GCNFs } & $\mathrm{C} 1 \mathrm{~s}$ & 285.0 & 0.0 \\
& $\mathrm{~N} 1 \mathrm{~s}$ & 400.0 & 89.8 \\
& $\mathrm{O} 1 \mathrm{~s}$ & 532.5 & 1.4 \\
& $\mathrm{~S} 2 \mathrm{p}$ & 164.7 & 0.7 \\
\hline \multirow{3}{*}{ Giprofloxacin-labeled } & $\mathrm{C} 1 \mathrm{~s}$ & 285.0 & 93.9 \\
& $\mathrm{~N} 1 \mathrm{~s}$ & 400.0 & 0.7 \\
& $\mathrm{O} 1 \mathrm{~s}$ & 533.6 & 5.3 \\
& $\mathrm{~S} 2 \mathrm{p}$ & 164.2 & 0.1 \\
\hline \multirow{3}{*}{ Tobramycin-labeled } & $\mathrm{C} 1 \mathrm{~s}$ & 284.8 & 91.4 \\
GCNFs & $\mathrm{N} 1 \mathrm{~s}$ & 399.6 & 0.8 \\
& $\mathrm{O} 1 \mathrm{~s}$ & 533.4 & 6.9 \\
& $\mathrm{~S} 2 \mathrm{p}$ & 164.2 & 0.9 \\
\hline
\end{tabular}

monitoring (MRM) ion transitions for amikacin, ciprofloxacin, and tobramycin (Table 1) were optimized by direct infusion of pure standards. MRMs were optimized on an Applied BioSystems 2000 (Framingham, MA) using electrospray ionization (ESI) with a Shimadzu Prominence HPLC system consisting of a Shimadzu LC-20 pump and SIL-20A autosampler (Shimadzu, Colombia, MD) operated under Analyst software control. Each antibiotic was prepared at a concentration of approximately $1 \mu \mathrm{g} \mathrm{mL}^{-1}$ in methanol $(0.1 \%$ formic acid) and infused at $10 \mu \mathrm{L} \mathrm{min}^{-1}$ into the LC mobile phase $\left(1.0 \mathrm{~mL} \mathrm{~min}^{-1}, 50 / 50\right.$ acetonitrile/water $(0.1 \%$ formic acid)) to optimize source and compound dependent parameters. Optimized positive ion mode ESI source parameters were as follows: curtain gas $(\mathrm{CUR})=20$ psi, CAD gas $=$ 5 , nebulizing gas $(\mathrm{GAS} 1)=60$, auxiliary gas $(\mathrm{GAS} 2)=$ 60 , source temperature $(\mathrm{TEM})=550^{\circ} \mathrm{C}$, and ESI voltage (IS) $=+5500 \mathrm{~V}$. For each antibiotic studied, the optimized compound declustering potential (DP) and collision energy (CE) are provided in Table 1 . The focusing potential (FP) was maintained at $+400 \mathrm{~V}$, the collision cell exit potential (CXP) at $+4 \mathrm{~V}$, and the $\mathrm{Q} 0$ entrance potential (EP) at $+10 \mathrm{~V}$ for all MRMs. The dwell time for each MRM transition during FI analysis was $500 \mathrm{~ms}$. Calibration curves were prepared that spanned the concentration range of $0.1-50 \mathrm{ppm}$ which was 
adequate for the quantities encountered in wash filtrates. Detection limits in parts-per-billion are defined at a signalto-noise ratio of three which are listed in Table 1.

2.7. Bioassay of Antibiotic-Labeled GCNFs. Pseudomonas aeruginosa was grown on LB medium overnight at $30^{\circ} \mathrm{C}$ with aeration. A $10 \mu \mathrm{L}$ sample of the growth was used to inoculate tubes containing $5 \mathrm{~mL}$ of LB broth. For the control, $100 \mu \mathrm{L}$ of sterile distilled water was added to one tube. A $100 \mu \mathrm{L}$ aliquot of test solution containing either tobramycin (108 ppm), amikacin (118 ppm), ciprofloxacin (100 ppm), $\mathrm{RuO}_{4}$-oxidized GCNFs (7.1 mg GCNFs in $2.0 \mathrm{~mL} \mathrm{H}_{2} \mathrm{O}$ ), tobramycin-labeled $\mathrm{RuO}_{4}$-oxidized GCNFs (7.9 mg GCNFs in $2.0 \mathrm{~mL} \mathrm{H} \mathrm{H}_{2} \mathrm{O}$ ), amikacin-labeled $\mathrm{RuO}_{4}$-oxidized GCNFs (7.9 mg GCNFs in $2.0 \mathrm{~mL} \mathrm{H}_{2} \mathrm{O}$ ), or ciprofloxacin-labeled $\mathrm{RuO}_{4}$-oxidized GCNFs (7.9 mg GCNFs in $2.0 \mathrm{~mL} \mathrm{H}_{2} \mathrm{O}$ ) was added to the other tubes, each containing $5 \mathrm{~mL}$ of LB broth. The resulting suspensions were then sonicated to achieve complete dissolution, typically $30 \mathrm{~min}$. All tubes were incubated at $37^{\circ} \mathrm{C}$ with shaking at $100 \mathrm{rpm}$ for $3 \mathrm{~h}$.

After the incubation period, serial dilutions were made for each sample and pour plates over a dilution range from $10^{0}$ to $10^{-9}$ were prepared using LB agar. The plates were incubated at $30^{\circ} \mathrm{C}$ overnight and the colonies were then counted to determine the number of viable cells present in each of the cultures. Serial dilution assays were conducted multiple times to verify reproducibility.

For OD600 measurements, Pseudomonas aeruginosa was grown on LB medium as described above, except that samples were incubated for $5.5 \mathrm{~h}$ at $37^{\circ} \mathrm{C}$ with shaking at $100 \mathrm{rpm}$. A Beckman DU 530 Life Science UV/Vis Spectrophotometer was calibrated and used to measure the optical density of $P$. aeruginosa of each sample at a fixed wavelength of $600 \mathrm{~nm}$ (OD600).

\section{Results and Discussion}

3.1. Covalent Functionalization and Characterization of GCNFs. Covalent, as opposed to noncovalent, methods were selected for the functionalization of GCNFs because it was expected that the covalent attachment of ligands would provide materials with greater stability. The leaching of antibiotics from the GCNF surface could create problems with bioassays in cell culture as well as unfavorable effects in the use of GCNFs as biomaterials due to the accumulation of antibiotics in certain tissue [21]. We considered that the covalent attachment of aminoglycoside antibiotics could be based on the reactions with surface oxides that are introduced by the treatment of GCNFs with an oxidant. Aldehyde and ketone groups, carboxylic acid and hydroxyl groups, as well as other surface oxides that are introduced serve as sites of covalent attachment of ligands, with the appropriate choice of coupling reactions. A necessary first step was the characterization of the surface of the oxidized GCNFs. We recently applied a method known as FLOSS [28-30] (fluorescence labeling of surface species) to the identification and quantification of surface oxides on nitric acid-oxidized herringbone GCNFs. In our study of graphitic carbon nanofibers, aldehyde/ketone, carboxyl, and hydroxyl groups were identified on as-produced, demineralized, and nitric acid-oxidized herringbone GCNFs by selective labeling reactions and quantified using FLOSS [31]. Since the surface oxides will serve as the sites of covalent attachment of antibiotics, it was useful to have this information in planning the labeling experiments of GCNFs with aminoglycoside antibiotics, as described in the following section.

3.2. GCNF-Labeling with Tobramycin by Reductive Amination. Aminoglycoside antibiotics were selected because their chemistry, biological properties, mechanism of action, and central role in antibacterial chemotherapy have been widely studied [32, 33]. Aminoglycosides have been used clinically against Pseudomonas aeruginosa infections. Another attractive feature was the presence of amino groups that would enable covalent attachment to either aldehyde/ketone groups by reductive amination or activated carboxylic acid groups by acylation. In the case of the aminoglycoside antibiotic tobramycin, the basicities of the five amino groups have been measured by $\mathrm{pH}$-dependent NMR and shown to be different [34], and this information was used to synthesize monoacylated analogs using substoichiometric amounts of a mild acylating agent and high dilution [35]. The products were those resulting from acylation at the primary amino group. Other studies of the synthesis of tobramycin analogs have shown that all five amino groups of the parent antibiotic can be acylated with $\mathrm{Cbz}$-chloride, using different conditions [36]. Amikacin would be expected to undergo labeling by reductive amination or acylation at either of its primary amino groups. Ciprofloxacin is one of the newest antibiotics of the fluoroquinolone class and possesses broad spectrum activity by a different mechanism of action [37]. Ciprofloxacin would be expected to undergo reaction selectively at its secondary amino group.

Our initial attempts at labeling nitric acid-oxidized GCNFs [31] with tobramycin were based on reductive amination, which can be carried out under mild reaction conditions and would be selective for aldehyde/ketone groups on the GCNF surface [38]. Tobramycin-labeled GCNFs 2 (Figure 3) were prepared by reductive amination using sodium borohydride. The resulting fibers were analyzed by FLOSS and contained low levels of antibiotic (0.12\%). The tobramycin-labeled GCNFs prepared by reductive amination were nonetheless tested for growth inhibition against Pseudomonas aeruginosa. Not surprisingly, no inhibition of bacterial growth was observed with these fibers (Figure 4). The plate count for cells in the control sample, the sample containing $\mathrm{RuO}_{4}$-oxidized GCNFs, and the sample containing tobramycin-labeled GCNFs were very similar, the colony counts being $9.5 \times 10^{4}, 4.0 \times 10^{4}$, and $75 \times 10^{4}$, respectively. These data indicate that viable colony formation is essentially the same in all three cases and that neither of the fiber samples possesses antibacterial activity. At this dilution, pure tobramycin completely inhibits growth as no surviving colonies are observed. While not encouraging, these initial experiments were nonetheless significant for two reasons. First, it became evident that higher levels of antibiotic incorporation would be necessary in order for GCNFs to be biologically active. 


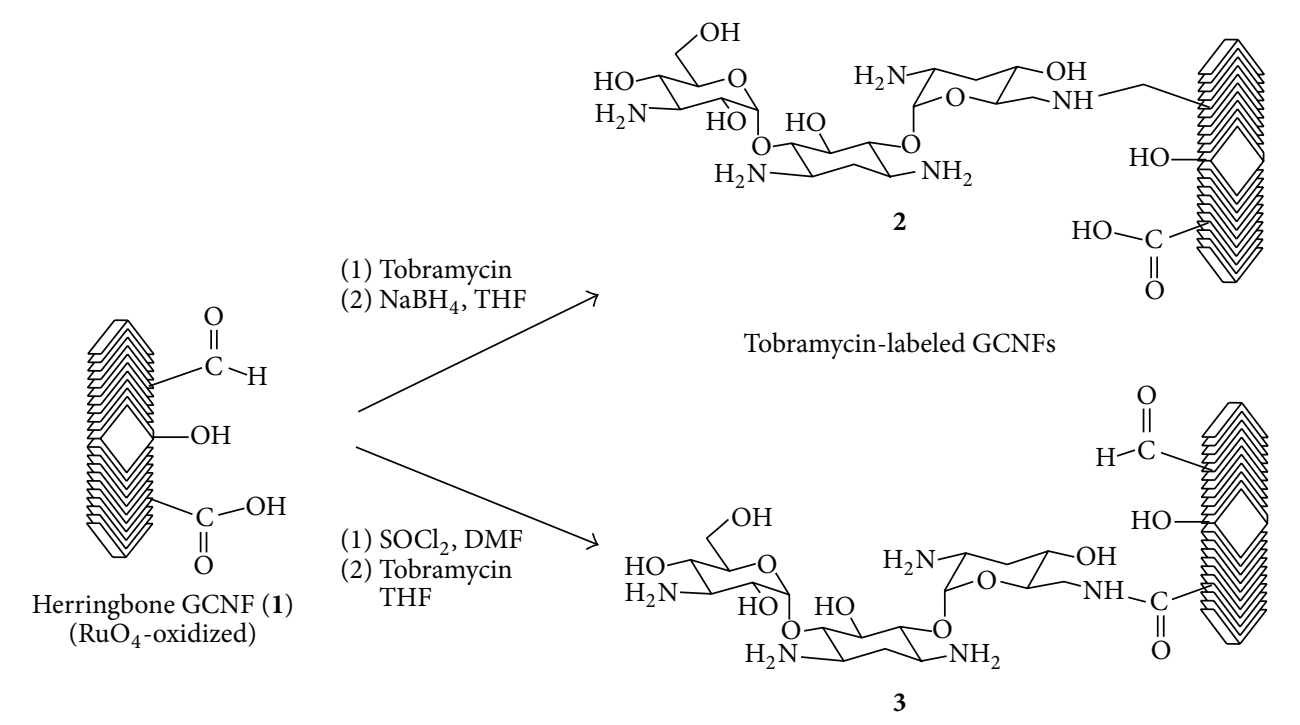

FIGURE 3: Labeling of oxidized herringbone GCNFs with tobramycin by reductive amination and by acylation.

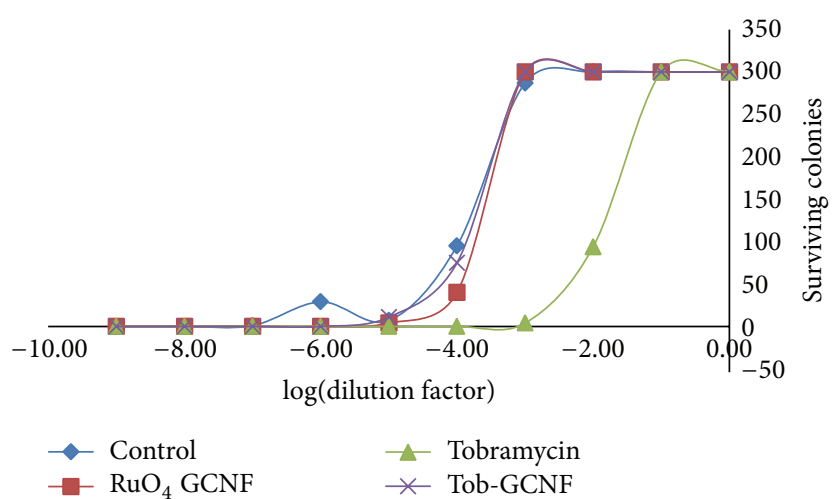

FIGURE 4: Plot of the number of surviving bacterial cell colonies as a function of the dilution factor on a logarithmic scale after $22 \mathrm{~h}$ incubation at $37^{\circ} \mathrm{C}$. $\mathrm{RuO}_{4}$-oxidized GCNFs were labeled with tobramycin by reductive amination.

Also, the chemical treatments used in oxidation and labeling do not in themselves confer inhibitory properties on the GCNFs; thus an important negative control emerged from these GCNF-labeling studies.

3.3. GCNF-Labeling by Thionyl Chloride Activation. We decided to reexamine GCNF-labeling in an effort to achieve higher levels of incorporation of antibiotic on the surface. We first explored an alternate method of oxidation to increase the presence of surface carbonyl-containing groups. Rasheed and coworkers had reported a study of the efficiency of the oxidation of carbon nanofibers with various oxidizing agents, including ruthenium tetroxide, which they report gave fibers containing higher amounts of carboxylic acids when compared to nitric acid or potassium permanganate [25]. During the course of our work in the use of oxidized GCNFs as catalysts for the reactions of 2-propanol [39], we investigated the use of other oxidants, using GCNFs of the herringbone morphology. We subjected herringbone GCNFs to oxidation with $\mathrm{RuO}_{4}$ and found that the fibers that were produced generally contained higher amounts of surface oxides than those produced using nitric acid. Carboxylic acid groups were the most abundant, at $4.71 \%$ of carbon in the GCNFs as determined by FLOSS [26]. It should be noted that $\mathrm{RuO}_{4}$ is catalytic in this procedure, with household bleach being present as the excess oxidant that reoxidizes insoluble $\mathrm{RuO}_{2}$ back to $\mathrm{RuO}_{4}$. Our initial attempts left residual ruthenium on the GCNFs, as evidenced by XPS. More frequent replenishing of the bleach that is added during the reaction and extensive washing gave oxidized GCNFs with no ruthenium detected by XPS.

For labeling of the carboxyl groups of the $\mathrm{RuO}_{4}$-oxidized herringbone GCNFs, we selected a method that is based on acylation of antibiotic with GCNF-derived acid chlorides. The fibers were first treated with thionyl chloride in $\mathrm{N}, \mathrm{N}$ - dimethylformamide, which converts surface carboxylic acid groups to acid chlorides, and the resulting fibers were treated with antibiotics in refluxing tetrahydrofuran (Figure 3). Using this convenient two-step sequence, tobramycin-, amikacin-, and ciprofloxacin-labeled GCNFs were synthesized from $\mathrm{RuO}_{4}$-oxidized fibers (Figure 5).

The only source of nitrogen in the GCNF-labeled fibers is from the antibiotics; thus XPS analysis allows quantification of each antibiotic in the functionalized GCNFs. Quantification in percent atom concentration of carbon, nitrogen, oxygen, and sulfur for $\mathrm{RuO}_{4}$-oxidized and antibioticlabeled GCNFs synthesized by the thionyl chloride activation method is shown in Table 2. From the percent atom concentrations of nitrogen, the molecular weights of the antibiotics, masses of the samples, and the percent by weight of antibiotic in the labeled fibers were calculated to be $11.7 \%$ amikacin in amikacin-labeled GCNFs, $5.4 \%$ ciprofloxacin in 


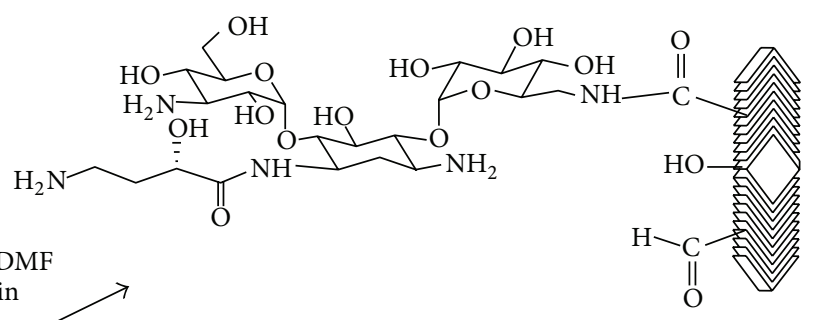

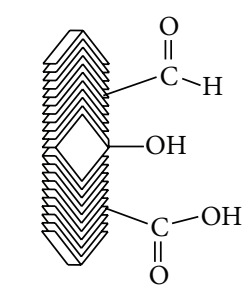

Herringbone GCNF 1
(1) $\mathrm{SOCl}_{2}, \mathrm{DMF}$

(2) Amikacin THF

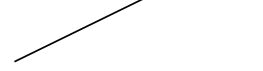

(1) $\mathrm{SOCl}_{2}, \mathrm{DMF} \longrightarrow$

(2) Ciprofloxacin THF

Amikacin-labeled GCNF 4

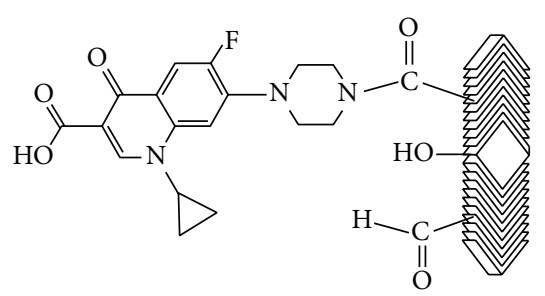

Ciprofloxacin-labeled GCNF 5

the ciprofloxacin-labeled GCNFs, and 5.3\% tobramycin in the tobramycin-labeled fibers.

3.4. SEM Images and Mass Spectrometric Analysis of Labeled GCNFs. One of the concerns was whether or not the GCNFs would survive the sequence of chemical steps necessary to activate the surface carboxyl groups and then label them with antibiotics. SEM images of $\mathrm{RuO}_{4}$-oxidized GCNFs and antibiotic-labeled GNFs are shown in Figure 6. The SEM images show that the graphite nanofibers have retained their structural integrity during both oxidation and labeling steps. Some clipping is apparent when these images are compared to as-produced herringbone GCNFs, but the fiber structure is relatively intact. Another concern was that the presence of unbound antibiotic retained by physisorption on the GCNF surface would severely affect the biological assays, since diffusion from the fiber surface would likely occur in the growth medium. LC-MS analysis of aqueous and organic washings of the fibers after labeling (see Section 2) indicated the presence of all three antibiotics in the filtrates. We were able to use mass spectrometry to aid in the development of a more efficient washing procedure to remove traces of antibiotics from the GCNFs after labeling. The labeled fibers were washed successively with dilute hydrochloric acid, water, dilute sodium bicarbonate solution, and then water. It was anticipated that the dilute $\mathrm{HCl}$ treatment would convert the amino groups to ammonium species, which would have greater aqueous solubility. After this series of washes, antibiotic was not detected in the filtrates obtained from any of the fibers by the LC-MS method, indicating that, if present, unbound antibiotic levels were below the limits of detection (Table 1).

3.5. Serial Dilution and Optical Density Assays of $\mathrm{RuO}_{4}$-Oxidized GCNFs Labeled by Acylation. Bactericidal activity of the three types of antibiotic-labeled GCNFs prepared by $\mathrm{RuO}_{4}$ oxidation and $\mathrm{SOCl}_{2}$ activation/amidation was evaluated by serial dilution experiments against Pseudomonas aeruginosa and presented in Figure 7, which shows the surviving colonies in each sample after the 3-hour incubation period. As shown in the figure, the control and $\mathrm{RuO}_{4}$-oxidized fibers have the same number of surviving colonies while the free antibiotics have the least number of surviving colonies as they are biocidal and kill the majority of the $P$. aeruginosa on the order of $96-99 \%$ of the bacteria as compared to the control. The amikacin-GCNFs, ciprofloxacin-GCNFs, and tobramycin-GCNFs have a similar number of surviving colonies and kill the P. aeruginosa on the order of $87-96 \%$ as compared to the control. While there are more colonies remaining in the antibiotic-labeled samples than in the samples with free antibiotic, there are significantly fewer colonies in the antibiotic-labeled GCNFs than in either the control or the $\mathrm{RuO}_{4}$-oxidized GCNFs, indicating that the antibiotics retain their biological activity.

Bacterial growth as indicated by colony forming units was also determined by optical density measurements (OD600) for Pseudomonas aeruginosa in the presence of unlabeled and antibiotic-labeled GCNFs. Data are presented in Figure 8. OD600 values were converted to colony forming units using the conversion factor of $4.5 \times 10^{5}$ colony forming units $(\mathrm{CFU}) / \mathrm{mL}$ for an OD of 1.0 obtained from the calibration. Consistent with the serial dilution assays, these data also show that colonies grown in the presence of $\mathrm{RuO}_{4}$-oxidized GCNFs have nearly identical growth to those grown in the control sample. As expected, samples that contain amikacin, ciprofloxacin, or tobramycin alone possess the lowest CFU/ $\mathrm{mL}$. Interestingly, samples that contain GCNFs labeled with these antibiotics have intermediate $\mathrm{CFU} / \mathrm{mL}$, showing decreases in cell density of approximately $40-50 \%$ relative to the $\mathrm{CFU} / \mathrm{mL}$ in the control $\left(3.59 \times 10^{5}\right)$ and $\mathrm{RuO}_{4}$-oxidized GCNFs $\left(3.69 \times 10^{5}\right)$. The decrease in cell density demonstrates 

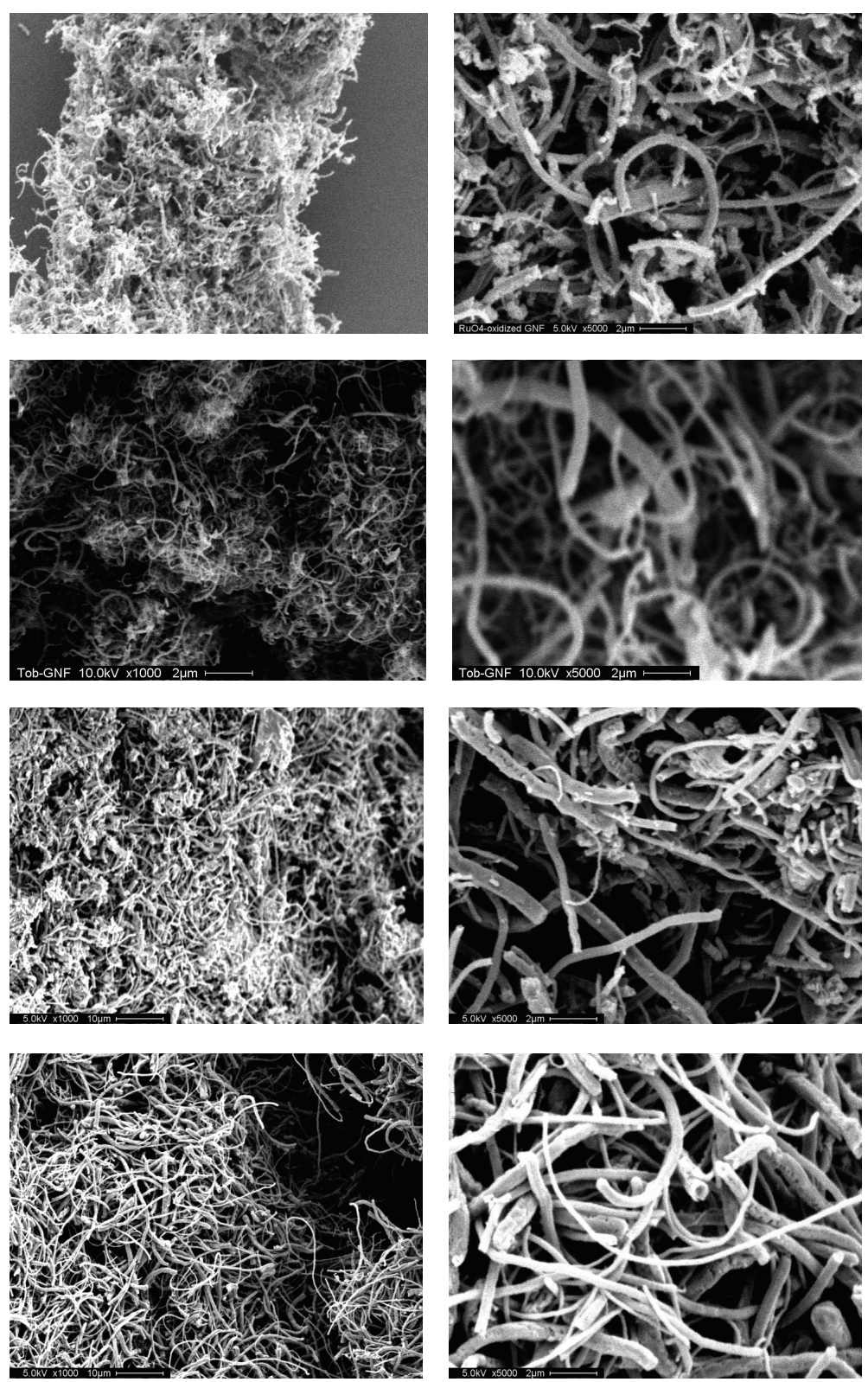

FiguRE 6: SEM images of $\mathrm{RuO}_{4}$-oxidized GNFs (top) and tobramycin-, amikacin-, and ciprofloxacin-labeled GNFs, at 1000x (left) and 5000x (right).

that the GCNFs labeled with antibiotics inhibit Pseudomonas aeruginosa growth, again to a lower extent than unbound antibiotic. The MIC for $50 \%$ inhibition of tobramycin against $P$. aeruginosa was determined to be $1 \mu \mathrm{g} / \mathrm{mL}(1 \mathrm{ppm})$ by Shawar et al. [40]. The concentration of GCNF-bound tobramycin used in the serial dilution and OD600 measurements in this study was $4.26 \mathrm{ppm}$. It is not surprising that higher levels of tobramycin are necessary to achieve inhibition when the drug is bound to the GCNF surface owing to the likely exclusion of the drug-GCNF conjugate from the cell interior where aminoglycosides are known to exert their primary mechanism of action (vide infra) [41].

\section{Conclusion}

Aminoglycoside antibiotics are used clinically to treat a wide range of bacterial infections that involve Gram-negative pathogens. Tobramycin is particularly effective against strains of Pseudomonas aeruginosa and has been used to treat infections of the eye, meningitis, and other infections. Amikacin is also active against $P$. aeruginosa and is used to treat severe mycobacterial and drug-resistant infections. Like other members of the aminoglycoside family of antibiotics, tobramycin and amikacin inhibit bacterial protein synthesis at the ribosomal level, by binding to the bacterial 30 S ribosomal subunit, 


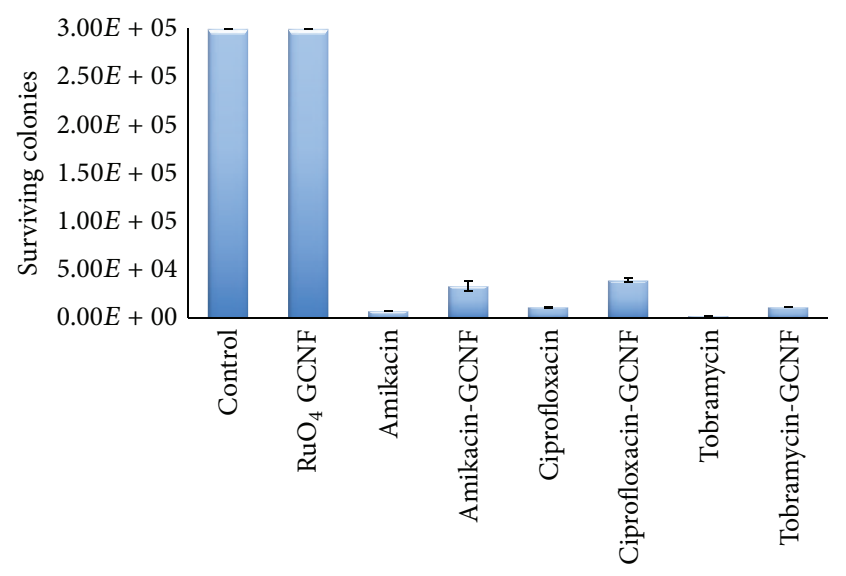

FIgURE 7: Bar graph showing surviving colonies of $P$. aeruginosa in the presence of no GCNFs, $\mathrm{RuO}_{4}$-oxidized GCNFs, and antibioticlabeled GCNFs.

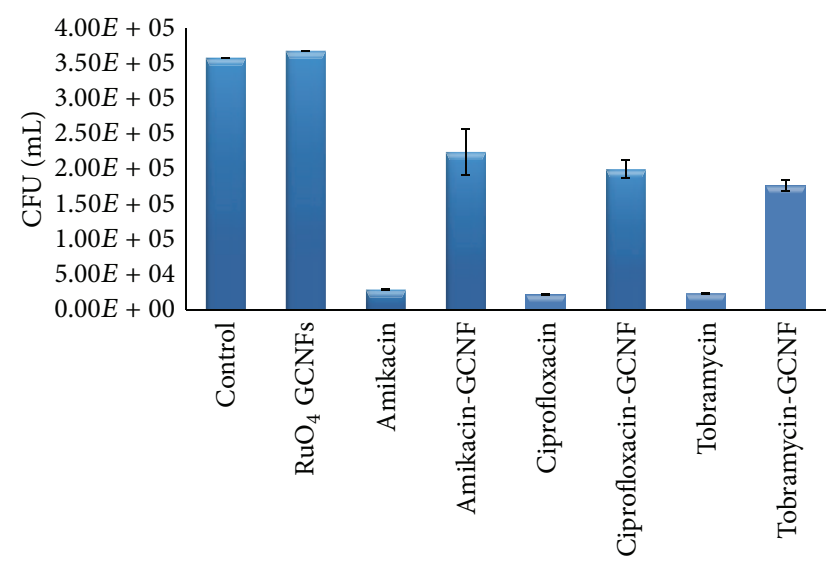

FIgURE 8: Colony forming units measured by OD600 of Pseudomonas aeruginosa in the presence of amikacin-, ciprofloxacin-, and tobramycin-labeled GCNFs.

resulting in a misreading effect [41]. Ciprofloxacin belongs to the fluoroquinolone class of antibiotics and possesses broad spectrum activity against Gram-positive and Gram-negative organisms. Ciprofloxacin acts by inhibiting the enzyme DNA gyrase, a topoisomerase that is essential for separation of bacterial DNA during cell division [42].

A question that we sought to address from the outset was whether or not the antibiotics used in this study would retain biocidal activity when bound to the graphitic surface. The serial dilution and OD600 assays for $\mathrm{RuO}_{4}$-oxidized GCNFs that have been labeled with tobramycin, amikacin, or ciprofloxacin all show inhibition of bacterial growth and demonstrate that these novel graphitic materials exhibit activity against $P$. aeruginosa. To our knowledge, these results represent the first examples of the synthesis of antibiotic-GCNF conjugates and demonstration of their antibacterial activity. GCNF-bound antibiotics show decreased activity when compared to the unbound drugs, perhaps the result of a difference in the mechanisms of action that operate when the drugs are bound to a surface.

The most widely cited mechanisms of action for all three antibiotics chosen in this study involve intracellular targets, which would seem inaccessible to these drugs when bound to GCNFs. Alternative mechanisms of biocidal activity, at least in the case of bound aminoglycosides, have been suggested in other studies. For example, it has been shown that a gentamicin-bovine serum albumin conjugate applied to gold nanoparticles exhibited biocidal activity against Pseudomonas aeruginosa [22]. The antibacterial activity of the gentamicin-BSA conjugate was ascribed to the disruption of the cell envelope due to ionic binding of the drug to the cell surface. Electron microscopy indicated that the conjugates were present on the cell surface and not inside the cell. In a different study, polycationic tobramycin-lipid conjugates were shown to be active against strains of Pseudomonas, and, again, a membranolytic mode of action was proposed involving the aminoglycoside antibiotic [43]. In the case of antibiotic-labeled GCNFs, we propose that the weakening of the cell envelope may be the result of a similar polycation effect in which protonated amino groups on the antibioticlabeled GCNFs interact with the cell surface. The results of our study suggest that surface-bound aminoglycosides, and also a fluoroquinolone, may possess an alternate mode of action that involves destabilization of the cell membrane. That antibiotic activity which is retained in these graphitic systems is an exciting prospect for further investigation of labeled GCNFs and for the potential use of graphitic carbonbased nanomaterials in biomedical applications. GCNFs functionalized with aminoglycosides may serve not only as scaffolds for new biomaterials, but also as probes for a ribosome or gyrase-specific versus a nonspecific membranolytic mechanism of action in these important classes of antibiotics. In summary, we have developed methods for the synthesis of graphite nanofibers that are covalently functionalized with antibiotics and we have shown that these novel materials possess antibacterial activity against $P$. aeruginosa. Further studies can now be carried out to determine inhibition against other organisms as well as biofilm formation and to investigate GCNFs that are labeled with other types of antibiotics.

\section{Conflict of Interests}

The authors declare that there is no conflict of interests regarding the publication of this paper.

\section{Acknowledgments}

The authors thank the Research Corporation and Villanova University for financial support. They also thank Dr. Randy Weinstein, Department of Chemical Engineering, Villanova University, for samples of herringbone GCNFs, and Drs. John Friede and James Wilson of the Department of Biology, Villanova University, for their assistance with microbiological experiments and Drs. Norman Dollahon and Ronald Balsamo of the Department of Biology, Villanova University, for their assistance with electron microscopy. 


\section{References}

[1] N. M. Rodriguez, A. Chambers, and R. T. K. Baker, "Catalytic engineering of carbon nanostructures," Langmuir, vol. 11, no. 10, pp. 3862-3866, 1995.

[2] M. S. Kim, N. M. Rodriguez, and R. T. K. Baker, "The interaction of hydrocarbons with coppernickel and nickel in the formation of carbon filaments," Journal of Catalysis, vol. 131, no. 1, pp. 6073, 1991.

[3] N. M. Rodriguez, M. S. Kim, and R. T. K. Baker, "Promotional effect of carbon monoxide on the decomposition of ethylene over an iron catalyst," Journal of Catalysis, vol. 144, no. 1, pp. 93-108, 1993.

[4] N. Krishnankutty, C. Park, N. M. Rodriguez, and R. T. K. Baker, "The effect of copper on the structural characteristics of carbon filaments produced from iron catalyzed decomposition of ethylene," Catalysis Today, vol. 37, no. 3, pp. 295-307, 1997.

[5] C. Park, E. S. Engel, A. Crowe, T. R. Gilbert, and N. M. Rodriguez, "Use of carbon nanofibers in the removal of organic solvents from water," Langmuir, vol. 16, no. 21, pp. 8050-8056, 2000.

[6] C. A. Bessel, K. Laubernds, N. M. Rodriguez, and R. T. K. Baker, "Graphite nanofibers as an electrode for fuel cell applications," Journal of Physical Chemistry B, vol. 105, no. 6, pp. 1115-1118, 2001.

[7] A. Chambers, C. Park, R. T. K. Baker, and N. M. Rodriguez, "Hydrogen storage in graphite nanofibers," Journal of Physical Chemistry B, vol. 102, no. 22, pp. 4253-4256, 1998.

[8] P. Serp, M. Corrias, and P. Kalck, "Carbon nanotubes and nanofibers in catalysis," Applied Catalysis A: General, vol. 253, no. 2, pp. 337-358, 2003.

[9] J. Wang and Y. Lin, "Functionalized carbon nanotubes and nanofibers for biosensing applications," Trends in Analytical Chemistry, vol. 27, no. 7, pp. 619-626, 2008.

[10] B. L. Fletcher, E. D. Hullander, A. V. Melechko et al., "Microarrays of biomimetic cells formed by the controlled synthesis of carbon nanofiber membranes," Nano Letters, vol. 4, no. 10, pp. 1809-1814, 2004.

[11] T. E. McKnight, A. V. Melechko, B. L. Fletcher et al., "Resident neuroelectrochemical interfacing using carbon nanofiber arrays," Journal of Physical Chemistry B, vol. 110, no. 31, pp. 15317-15327, 2006.

[12] P. A. Tran, L. Zhang, and T. J. Webster, "Carbon nanofibers and carbon nanotubes in regenerative medicine," Advanced Drug Delivery Reviews, vol. 61, no. 12, pp. 1097-1114, 2009.

[13] L. Li, J. Li, and C. M. Lukehart, "Graphitic carbon nanofiberpoly(acrylate) polymer brushes as gas sensors," Sensors and Actuators B: Chemical, vol. 130, no. 2, pp. 783-788, 2008.

[14] X. Wang, O. Ramström, and M. Yan, "Glyconanomaterials: synthesis, characterization, and ligand presentation," Advanced Materials, vol. 22, no. 17, pp. 1946-1953, 2010.

[15] Y. Lin, S. Taylor, H. Li et al., "Advances toward bioapplications of carbon nanotubes," Journal of Materials Chemistry, vol. 14, no. 4, pp. 527-541, 2004.

[16] Y. M. Chabre and R. Roy, "Design and creativity in synthesis of multivalent neoglycoconjugates," in Advances in Carbohydrate Chemistry and Biochemistry, D. Horton, Ed., vol. 63, pp. 165393, 2010

[17] D. Nepal, S. Balasubramanian, A. L. Simonian, and V. A. Davis, "Strong antimicrobial coatings: single-walled carbon nanotubes armored with biopolymers," Nano Letters, vol. 8, no. 7, pp. 18961901, 2008.
[18] B. Ratner, A. Hoffman, F. Schoen, and J. Lemons, Eds., Biomaterials Science: An Introduction to Materials in Medicine, Elsevier, Amsterdam, The Netherlands, 2004.

[19] R. M. Donlan, "Biofilm formation: a clinically relevant microbiological process," Clinical Infectious Diseases, vol. 33, no. 8, pp. 1387-1392, 2001.

[20] P. Y. Litzler, L. Benard, N. Barbier-Frebourg et al., "Biofilm formation on pyrolytic carbon heart valves: influence of surface free energy, roughness, and bacterial species," Journal of Thoracic and Cardiovascular Surgery, vol. 134, no. 4, pp. 1025-1032, 2007.

[21] K. Vasilev, J. Cook, and H. J. Griesser, "Antibacterial surfaces for biomedical devices," Expert Review of Medical Devices, vol. 6, no. 5, pp. 553-567, 2009.

[22] J. L. Kadurugamuwa, A. J. Clarke, and T. J. Beveridge, "Surface action of gentamicin on Pseudomonas aeruginosa," Journal of Bacteriology, vol. 175, no. 18, pp. 5798-5805, 1993.

[23] A. Tiraferri, C. D. Vecitis, and M. Elimelech, "Covalent binding of single-walled carbon nanotubes to polyamide membranes for antimicrobial surface properties," ACS Applied Materials and Interfaces, vol. 3, no. 8, pp. 2869-2877, 2011.

[24] A. S. Kumar, S. Sornambikai, L. Deepika, and J.-M. Zen, "Highly selective immobilization of amoxicillin antibiotic on carbon nanotube modified electrodes and its antibacterial activity," Journal of Materials Chemistry, vol. 20, no. 45, pp. 10152-10158, 2010.

[25] A. Rasheed, J. Y. Howe, M. D. Dadmun, and P. F. Britt, "The efficiency of the oxidation of carbon nanofibers with various oxidizing agents," Carbon, vol. 45, no. 5, pp. 1072-1080, 2007.

[26] C. L. Stevens and C. P. Bryant, "Oxidation with ruthenium dioxide and hypochlorite," in Methods in Carbohydrate Chemistry, R. L. Whistler and J. N. BeMiller, Eds., vol. 6, pp. 337-341, 1972.

[27] F. Pompeo and D. E. Resasco, "Water solubilization of single-walled carbonnanotubes byfunctionalization with glucosamine," Nano Letters, vol. 2, no. 4, pp. 369-373, 2002.

[28] Y. Xing and E. Borguet, "Specificity and sensitivity of fluorescence labeling of surface species," Langmuir, vol. 23, no. 2, pp. 684-688, 2007.

[29] X. Feng, N. Dementev, W. Feng, R. Vidic, and E. Borguet, "Detection of low concentration oxygen containing functional groups on activated carbon fiber surfaces through fluorescent labeling," Carbon, vol. 44, no. 7, pp. 1203-1209, 2006.

[30] N. Dementev, X. Feng, and E. Borguet, "Fluorescence labeling and quantification of oxygen-containing functionalities on the surface of single-walled carbon nanotubes," Langmuir, vol. 25, no. 13, pp. 7573-7577, 2009.

[31] T. Pellenbarg, N. Dementev, R. Jean-Gilles et al., "Detecting and quantifying oxygen functional groups on graphite nanofibers by fluorescence labeling of surface species," Carbon, vol. 48, no. 15, pp. 4256-4267, 2010.

[32] D. P. Arya, Aminoglycoside Antibiotics: from Chemical Biology to Drug Discovery, John Wiley \& Sons, Hoboken, NJ, USA, 2007.

[33] K. L. Rinehart Jr. and T. Suami, Eds., Aminocyclitol Antibiotics, American Mathematical Society, Washington, DC, USA, 1979.

[34] K. F. Koch, J. A. Rhoades, E. W. Hagaman, and E. Wenkert, "Carbon-13 nuclear magnetic resonance spectroscopy of naturally occurring substances. XXV. Carbon-13 nuclear magnetic resonance spectral analysis of tobramycin and related antibiotics," Journal of the American Chemical Society, vol. 96, no. 10, pp. 3300-3305, 1974. 
[35] P. Singh, M. Pirio, D. K. Leung, and Y.-G. Tsay, "Controlled coupling of aminoglycoside antibiotics to proteins for use in homogeneous enzyme immunoassays," Canadian Journal of Chemistry, vol. 62, no. 11, pp. 2471-2477, 1984.

[36] S. Hanessian, M. Tremblay, and E. E. Swayze, "Tobramycin analogues with C-5 aminoalkyl ether chains intended to mimic rings III and IV of paromomycin," Tetrahedron, vol. 59, no. 7, pp. 983-993, 2003.

[37] K. Drlica and X. Zhao, "DNA gyrase, topoisomerase IV, and the 4-quinolones," Microbiology and Molecular Biology Reviews, vol. 61, no. 3, pp. 377-392, 1997.

[38] A. B. Reitz and E. W. Baxter, "Reductive aminations of carbonyl compounds with borohydride and borane reducing agents," Organic Reactions, vol. 59, pp. 1-714, 2004.

[39] A. R. Ferens, R. D. Weinstein, R. M. Giuliano, and J. A. Hull, "Selective decomposition of isopropanol using as prepared and oxidized graphite nanofibers," Carbon, vol. 50, no. 1, pp. 192200, 2012.

[40] R. M. Shawar, D. L. MacLeod, R. L. Garber et al., "Activities of tobramycin and six other antibiotics against Pseudomonas aeruginosa isolates from patients with cystic fibrosis," Antimicrobial Agents and Chemotherapy, vol. 43, no. 12, pp. 2877-2880, 1999.

[41] B. D. Davis and P.-C. Tai, "Multiple interactions of aminoglycoside antibiotics with ribosomes," in Aminocyclitol Antibiotics, K. L. Rinehart Jr. and T. Suami, Eds., pp. 413-422, American Chemical Society, Washington, DC, USA, 1979.

[42] R. J. Reece and A. Maxwell, "DNA gyrase: structure and function," Critical Reviews in Biochemistry and Molecular Biology, vol. 26, no. 3-4, pp. 335-375, 1991.

[43] R. Dhondikubeer, S. Bera, G. G. Zhanel, and F. Schweizer, "Antibacterial activity of amphiphilic tobramycin," Journal of Antibiotics, vol. 65, no. 10, pp. 495-498, 2012. 

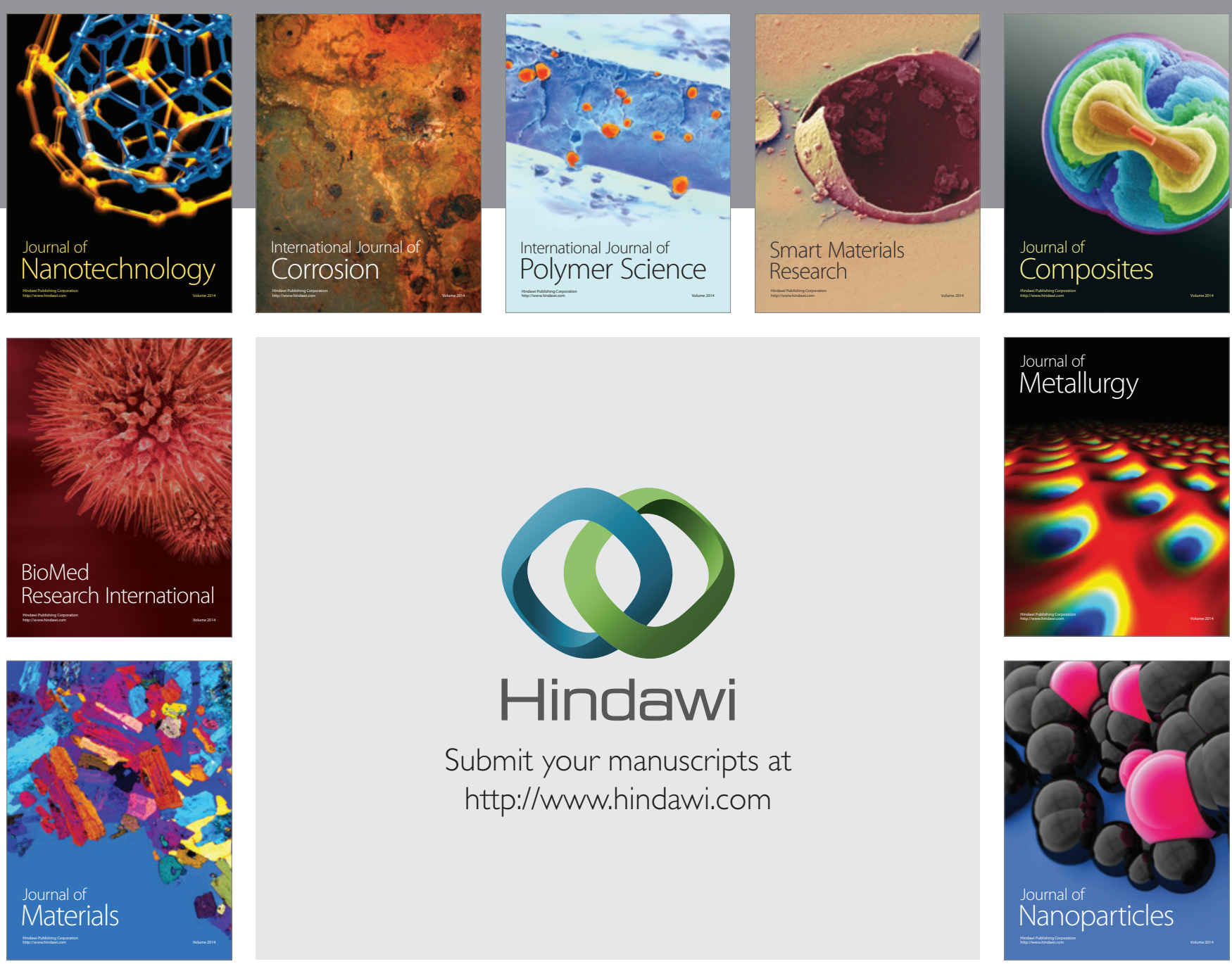

Submit your manuscripts at http://www.hindawi.com
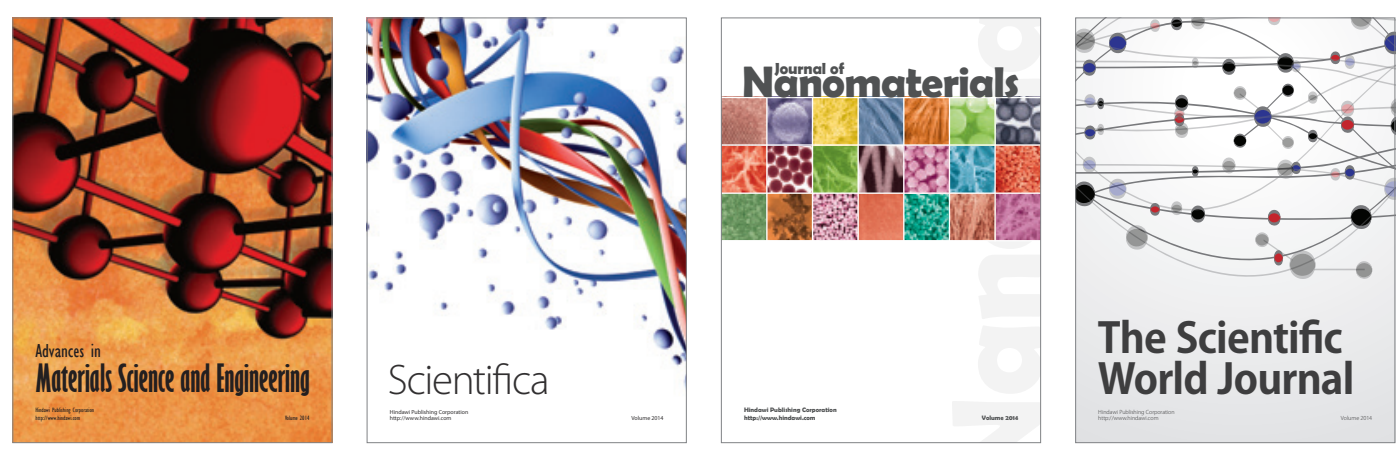

\section{The Scientific World Journal}
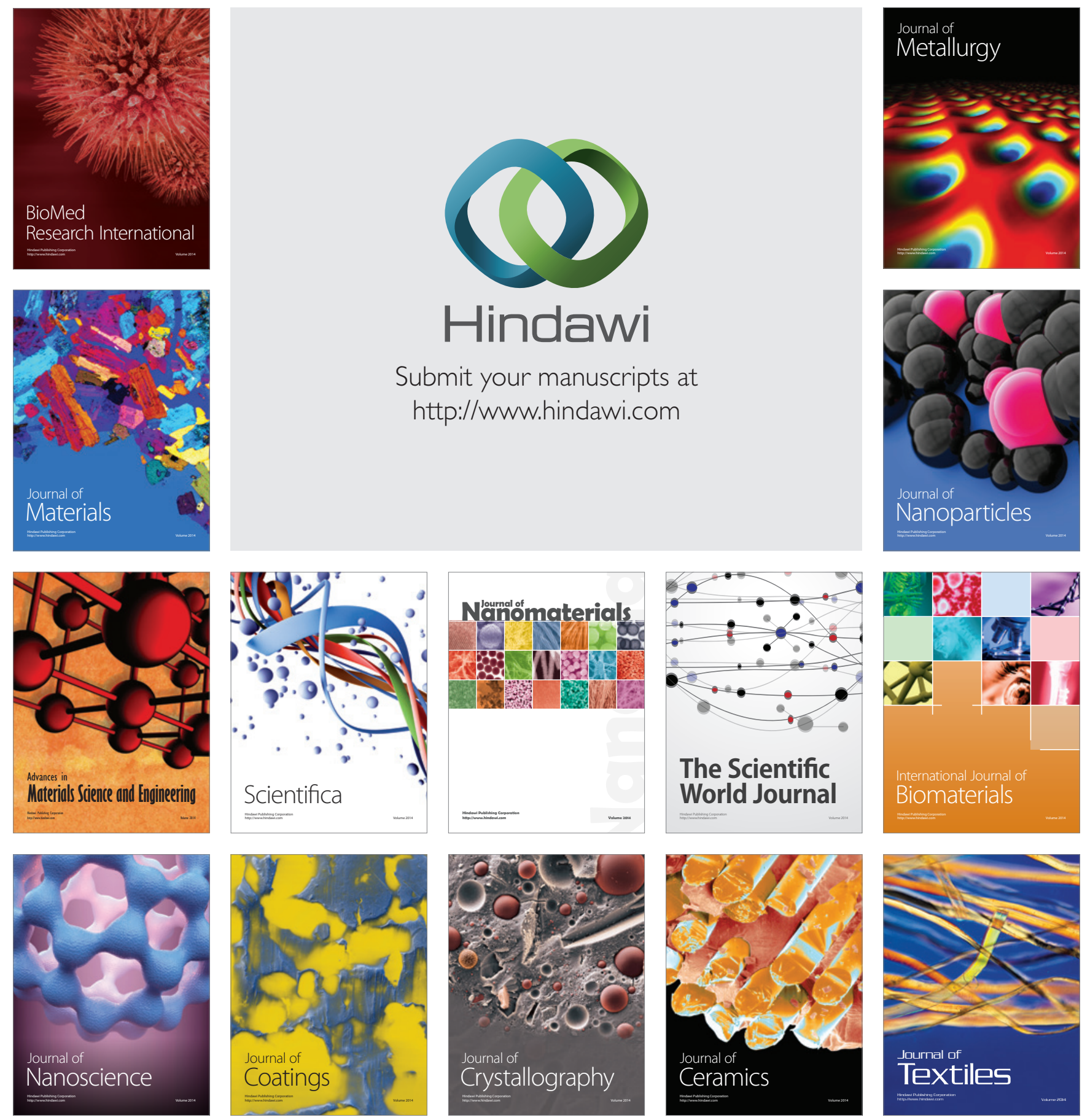